Escuela de Relaciones Internacionales.

Universidad Nacional, Costa Rica.

N. ${ }^{\circ} 90.1$ • Enero-Junio de 2017

doi: http://dx.doi.org/10.15359/ri.90-1.1

Pp. 15-46

\title{
ANÁLISIS ARANCELARIO DE PRODUCTOS SENSIBLES DE COSTA RICA EN LA NEGOCIACIÓN DE ACUERDOS COMERCIALES PREFERENCIALES
}

\author{
TARIFF ANALYSIS OF SENSITIVE PRODUCTS \\ OF COSTA RICA ON THE NEGOTIATION \\ OF PREFERENTIAL TRADE AGREEMENTS
}

\author{
Susana Wong Chan* \\ Duayner Salas Chaverri**
}

\section{RESUMEN:}

La reducción arancelaria ha sido el motivo principal para la negociación de acuerdos comerciales preferenciales, los que en la actualidad han tenido un incremento considerable. Siendo que las tendencias mundiales en aranceles aplicados tienden a la baja, los países aún conservan productos sensibles que no son objetivos de liberalización. Costa Rica no ha escapado de esta lógica, al poseer numerosos acuerdos comerciales a la vez que excluye ciertos productos del trato preferencial. El presente artículo, en primera instancia, valida tendencias mundiales en la negociación de acuerdos comerciales con las tendencias de Costa Rica. En segundo lugar, compara los aranceles consolidados y los aranceles aplicados en Costa Rica, determinando patrones entre sus brechas. Por último, identifica variables para la determinación de productos sensibles y analiza estos productos sensibles con respecto al tratamiento dentro de los distintos acuerdos comerciales firmados por Costa Rica.

Palabras claves: Arancel; productos sensibles; política arancelaria; comercio internacional; comercio; libre comercio; Costa Rica.

\begin{abstract}
:
Tariff reduction has been the main reason for the negotiation of preferential trade agreements that have had been having a substantial increase nowadays. Despite the fact that the applied tariff have been decreasing according to the world tendencies, the countries still preserve sensitive products without any liberalization. Costa Rica is within this logic, because it has numerous trade agreements and at the same time excludes some products from the preferential treatment. This paper, in the first instance, validates world tendencies regarding the negotiation of trade agreements with the tendencies of Costa Rica. In second place, it compares bound tariffs and the applied tariffs in Costa Rica, determining patterns between their gaps. At last, it identifies variables for the determination of sensitive products regarding the treatment inside the trade agreements signed by Costa Rica.
\end{abstract}

Costa Rica has a trade agreement were negotiated with tariff reduction interest or not.

Keywords: Tariff; Sensitive Products; Customs Policy; International Trade; Trade; Free Trade; Costa Rica.

* Máster. Coordinadora, docente e investigadora de la carrera de Administración Aduanera y Comercio Exterior. Escuela de Administración Pública. Universidad de Costa Rica. Correo electrónico: Susana.wong@ucr.ac.cr

** Máster. Docente e investigador de la carrera de Administración Aduanera y Comercio Exterior. Escuela de Administración Pública. Universidad de Costa Rica. Correo electrónico: duayner.salas@ucr.ac.cr 


\section{Introducción}

Un tratado de libre comercio (TLC) constituye una herramienta utilizada para crear una zona de libre comercio (ZLC). El artículo XXIV b) del GATT define una ZLC como:

Un grupo de dos o más territorios aduaneros entre las cuales se eliminan los derechos de aduanas y las demás reglamentaciones comerciales restrictivas... con respecto a lo esencial de los intercambios comerciales de los productos originarios de los territorios constitutivos de dicha zona de libre comercio. (OMC, 1994, p. 547)

Tal y como se aprecia en la definición anterior, uno de los puntos elementales de un TLC, en la actualidad, es la eliminación de derechos arancelarios ${ }^{1}$ La negociación de TLC tal y como se conoce hoy, inició con la apertura y liberalización de Gran Bretaña en el siglo XIX. Durante esa época, el Imperio Británico decide eliminar sus aranceles de manera unilateral y firmar el tratado Cobden-Chavalier, en 1860, con Francia. Este tratado tiene como fin reducir aranceles y crear un ambiente de nación más favorecida ${ }^{2}(\mathrm{NMF})$ entre ellos. Gracias al tratado Cobden-Chavalier, se desató un "efecto dominó" que impulsó la negociación de otros acuerdos bilaterales entre otras potencias europeas para conseguir acceso equivalente a los concedidos en el Cobden-Chavalier. Esta oleada permitió que, en Europa, se redujeran los aranceles a un 50\% (OMC, 2011b, p. 49).

Tras un gran impulso en el establecimiento de acuerdos comerciales, a principios del siglo XX, estallan varias guerras comerciales aisladas y la I Guerra Mundial. Estas guerras ocasionaron un freno en la creación de normas comerciales conjuntas. Se dieron intentos para restablecer la cooperación económica -por ejemplo, la Conferencia Económica Mundial organizada por la Sociedad de las Naciones en 1920- pero ninguna fue fructífera. Aunado a lo anterior, en 1930 estalla la Gran Depresión y posteriormente la II Guerra Mundial. Ambos eventos ocasionan que los países elevaran los obstáculos a la importación y, por ende, erosionaran los avances obtenidos en años anteriores (OMC, 2011b).

Con el fin de evitar acontecimientos como los previamente descritos, las potencias mundiales de la época -Estados Unidos y Gran Bretaña- coinciden en que

1 Los derechos arancelarios son "los gravámenes que deben soportar las mercancías cuando son objeto de comercio entre dos países que atraviesan la frontera" (Giler et al, 2010, p. 3).

2 Nación más favorecida debe ser entendida en el sentido de que toda ventaja otorgada por un miembro de la OMC debe ser aplicado, tal cual y sin discriminación alguna, a todos los demás miembros de la OMC. Para más información ver el Artículo I del GATT. 
era necesario construir un nuevo sistema económico internacional para evitar la inestabilidad financiera y rivalidad de bloques comerciales o de países. De esta forma, en la Conferencia de Bretton Woods de 1944, se decide crear tres nuevas instituciones de las cuales únicamente el Fondo Monetario Internacional (FMI) y el Banco Mundial (BM) fueron formalmente constituidas. La otra institución denominada Organización Internacional del Comercio (OIC) no fue conformada, en su lugar, se creó una institución de facto a través de la adopción del Acuerdo General sobre Aranceles Aduaneros y Comercio de 1947 (GATT 1947) por diversos países. Desde 1947 se efectuaron 8 rondas de negociación para, entre otras cosas, reducir los aranceles de importación. La última ronda de negociación se llevó a cabo entre 1986 y 1994 y finalizó con la creación de la Organización Mundial del Comercio (OMC). Con la creación de la OMC, todos los miembros tuvieron que consolidar sus aranceles. El proceso de consolidación de los aranceles se refiere al proceso por el cual cada miembro de la $\mathrm{OMC}$ se compromete a no elevar su arancel aplicado ${ }^{3}$ a la importación sobre la cifra consolidada en su lista de acceso (OMC, 2011b).

Dicho proceso de consolidación fue un gran paso hacia la reducción arancelaria mundial. El recorte paulatino de los aranceles consolidados durante las diferentes rondas de negociaciones, así como la disminución del arancel aplicado por Costa Rica y otros países, han reducido las posibilidades para obtener preferencias arancelarias significativas en un TLC. Según datos de la OMC, el promedio mundial del arancel aplicado es de un $4 \%$, por lo tanto, se afirma que la posibilidad de obtener preferencias arancelarias significativas en un TLC ha sido reducida (OMC, 2011b).

A pesar de que las posibilidades de obtener preferencias arancelarias son bajas, la OMC, con sus 164 miembros (al 29 de julio del año 2016), no ha frenado la negociación y firma de tratados. De hecho, para el 01 de julio del 2016, se notificaron 635 acuerdos comerciales regionales (ACR) de los cuales, un $90 \%$ corresponde a TLC y acuerdos de alcance parcial y un $10 \%$ corresponde a uniones aduaneras (OMC, 2016). Esto se debe a que parcialmente hay otros motivos por los cuales se negocian tratados y a que cada país posee un conjunto de productos sensibles a los que aún designa un arancel superior al $4 \%$ correspondiente al promedio mundial del arancel aplicado y, por ende, un TLC permite obtener acceso a mercados en estos casos.

Siendo que las tendencias mundiales en aranceles aplicados tienden a la baja, los países aún conservan productos sensibles que no son objeto de liberalización.

3 El arancel aplicado se refiere al derecho que se aplica efectivamente al momento de la importación de la mercancía. Este arancel puede ser inferior o igual al arancel consolidado ante la OMC, pero no puede ser superior. 
Según la tendencia mundial, productos agrícolas, textiles y sus derivados son productos sensibles y, por tanto, sujetos a altos niveles de aranceles.

Costa Rica no ha escapado de esta lógica. Actualmente el país tiene 14 TLC vigentes. Sin embargo, nunca se ha hecho un estudio para determinar cuáles han sido los productos sensibles en Costa Rica en el marco de las negociaciones comerciales. Esto es de gran relevancia, ya que permitirá determinar, si el país sigue las tendencias a nivel mundial, así como explorar en investigaciones subsecuentes las razones por las cuales negocia un TLC.

\section{Materiales y métodos}

El diseño de la investigación de este artículo es de carácter descriptivo y exploratorio, ya que busca, primero, verificar si efectivamente, en el caso de Costa Rica, se cumplen las tendencias mundiales en la negociación de acuerdos comerciales. Segundo, pretende listar y evidenciar las características de las mercancías que se definen como sensibles en Costa Rica. Este segundo aspecto, como principal resultado y aporte de la investigación.

Para indagar en el objeto de investigación, se aplica un enfoque mixto. Este enfoque mixto permite recabar información cuantitativa y cualitativa para obtener un mayor entendimiento de los acuerdos comerciales negociados por Costa Rica y, además, otorga mayor validez a los hallazgos.

La recolección de la información se basará en la sistematización de datos obtenidos a través de fuentes primarias y secundarias, a saber:

1. Porcentajes de aranceles aplicados del año 2014 y aranceles consolidados 2002 de todo el universo arancelario, obtenidos del COMEX y de la OMC.

2. Estadísticas generales de Costa Rica obtenidas de COMEX, entre 2013-2015.

3. Informes oficiales e investigaciones varias de la $\mathrm{OMC}$, ubicadas entre 2013-2015.

La investigación toma en cuenta una serie de variables independientes, y las relaciona con la variable dependiente que se define en virtud de la condición de mercancía sensible. Dentro de las variables independientes estudiadas se pueden mencionar arancel consolidado, arancel aplicado elevado, contingente 
arancelario elevado, condición de grano básico, condición de producción nacional y condición de industria con influencia política.

En el desarrollo de los resultados, se detalla, específicamente, a qué se hace referencia con conceptos especializados, así como los principales indicadores y fórmulas. En la mayoría de los casos, se utiliza modelaje y construcciones metodológicas a partir de los trabajos ya elaborados por la OMC.

\section{Resultados}

\section{Tendencias con respecto a productos sensibles}

El arancel promedio aplicado a nivel mundial es de aproximadamente un $4 \%$ y, por ende, únicamente un pequeño porcentaje de esos países gozan de un mayor margen de preferencias arancelarias. Sin embargo, se observará que hay productos que gozan de mayores preferencias, dependiendo del producto que se desea comercializar.

Productos sensibles se refieren a "aquellos que si fueran afectados por la liberalización podrían originar descalabros productivos y sociales, ya que los productores locales no tendrían capacidad de competir con la invasión de productos... en sus mercados locales... [además] son bienes producidos dentro de un país que representa una imprescindible fuente de empleo e ingresos para las personas del mismo" (COM, 2010, párr.22). Según Acosta (2005, p. 82), mercancía sensible se define como "aquella mercancía que se encuentra sujeta a elevados aranceles o a una o varias restricciones no arancelarias, tales como cupos, permisos previos, entre otras, cuya internación ilegal al país puede generar elevados beneficios económicos y de fácil comercialización en mercados informales".

Independientemente de la definición que se le dé, la sensibilidad de los productos depende de cada país, ya que radica en el tipo de producción que tiene una economía. En la mayoría de los casos, los siguientes productos son considerados sensibles: azúcar, arroz, prendas de vestir, productos lácteos, productos pesqueros, entre otros. Por ejemplo, Singapur no tiene realmente algún producto sensible, ya que su fuerte es en el comercio de servicios, por lo tanto, los aranceles aplicados a todos los productos son de $0 \%$. Sin embargo, países como Mauricio, en donde producen mercancías como azúcar, arroz, prendas de vestir y otros productos agrícolas, aplican un arancel elevado. A la vez, desde la perspectiva de las exportaciones, países como Singapur, que 
no produce bienes, tiene un margen preferencial muy bajo. Sin embargo, según el estudio de la $\mathrm{OMC}$, las mercancías exportables de Mauricio al mundo poseen un margen preferencial bastante elevado, aproximadamente un 24\% (OMC, 2011b).

Lo anterior también se sustenta con el hecho de que las exportaciones de productos procedentes de países menos adelantados (PMA) gozan de preferencias arancelarias más elevadas. Según el análisis de la OMC, el 16\% de las importaciones provenientes de los PMA tiene un margen preferencial superior al $10 \%$. Este $16 \%$ con márgenes preferenciales superiores al $10 \%$ se contrapone a lo indicado en el apartado anterior -que indica que solo un $2 \%$ tiene márgenes superiores al $10 \%-$.

Los PMA pagan, en promedio, aranceles más elevados que otros países y, por lo tanto, también gozan de mayores tasas preferenciales. Esto se debe a que generalmente los PMA producen bienes que son catalogados como sensibles. De hecho, según el indicador denominado tasa de utilización de las preferencias (TUP), se aplican más las preferencias arancelarias derivadas en un TLC para productos sensibles, ya que los beneficios son altos; caso contrario con respecto a los productos con márgenes preferenciales pequeños, porque las empresas deben incurrir en gastos para obtener beneficios que, a la larga, no son suficientes como para cubrir el gasto inicial del cumplimiento de las reglas de origen ${ }^{4}$ (OMC, 2011b).

Para poder determinar los productos sensibles de Costa Rica en las negociaciones comerciales, así como corroborar si se cumple o no con las tendencias mundiales, se requiere de un análisis arancelario de Costa Rica.

\section{Análisis arancelario de Costa Rica}

Todo producto importado a Costa Rica debe pagar los aranceles a la importación y otros impuestos, salvo que estén en $0 \%$ o sean parte de alguna excepción -como, por ejemplo, los uniformes escolares-. Los aranceles y los impuestos tienen un significado diferente. Los aranceles son los derechos aplicados únicamente a las mercancías importadas a Costa Rica y, como miembro de la OMC, se debe aplicar sobre la base NMF, es decir, no puede discriminar el nivel arancelario entre los miembros de la OMC. Cabe destacar que el arancel es el único derecho que se negocia en los TLC, mientras que los impuestos internos no

4 Las reglas de origen pueden ser definidas como "las disposiciones específicas desarrolladas a partir de los principios establecidos por la legislación nacional o por convenios internacionales ("criterios de origen'), aplicados por un país a fin de determinar el origen de las mercancías” (OMA, s. f., p. 6). 
lo son. En Costa Rica, el arancel es denominado derecho arancelario a la importación (DAI) y es aplicado sobre el valor aduanero $\mathrm{CIF}^{5}$ de las mercancías. Además del DAI, Costa Rica tiene también un derecho de $1 \%$ creado con la Ley N.o 6946 o Ley de Emergencia Nacional. Dicho 1\% de la Ley N.o 6946 también es aplicado a todos los productos importados salvo algunas excepciones ${ }^{6}$. Esta Ley tiene cierta particularidad, ya que el $1 \%$ de esta fue separado del DAI para, entre otras cosas, generar ingresos para defender el país en caso de alguna emergencia nacional. Por lo tanto, el 1\% de Ley N.o 6946 también es negociado dentro del marco de un TLC.

De esta manera, con la Resolución N. ${ }^{\circ}$ 48-94 (CONSEJO-XII) de 23-2-94, el Consejo de Ministros de Integración Económica Centroamericana (COMIECO) le autoriza a Costa Rica para que las tarifas de $10 \%$ y $15 \%$ incluyan el gravamen del 1\% de la Ley N.o 6946. Por tanto, a los incisos con tarifas de $10 \%$ y $15 \%$ se les debe aplicar un DAI de $9 \%$ y $14 \%$, respectivamente. A la vez, según el Decreto Ejecutivo N. ${ }^{\circ}$ 27246-COMEX-MEIC, todos los medicamentos incluidos dentro de las partidas 30.03 y 30.04 del sistema armonizado de designación y codificación de mercancías (SA) ${ }^{7}$, se le debe agregar un $1 \%$ por costo de internación y un 1\% por concepto de impuesto de la Ley N.o 6946.

En Costa Rica también se aplican otros impuestos calculados en el momento de la importación de las mercancías. Estos impuestos son: selectivo de consumo, impuesto de ventas, Instituto de Desarrollo Agrario (IDA), Instituto de Fomento y Asesoría Municipal (IFAM), impuesto específico y ganancia estimada. A pesar de que son calculados en el momento de la importación, estos impuestos aplican para absolutamente todas las mercancías que se comercialicen en lo interno de Costa Rica. Estos impuestos, al no ser negociados en el marco de un TLC, no serán considerados en este artículo.

Costa Rica es miembro de la OMC desde 1995, por lo tanto, desde ese entonces, ha consolidado sus aranceles ante dicha institución. Como se comentó anteriormente,

5 CIF es un término mundialmente conocido cuyo acrónimo está en inglés Cost, Insurance and Freight. En español se traduce como costo, seguro y flete.

6 Estas excepciones incluyen: (i) las importaciones abarcadas por los acuerdos de libre comercio, (ii) las mercancías que figuran en el Acuerdo de la OMC sobre tecnología de la información (véase (v) infra), (iii) las importaciones en las condiciones siguientes: (a) Régimen Zonas Francas de Exportación (Ley N.o 6695 del 10 de diciembre de 1981 y sus modificaciones), (b) Cooperativas de auto gestión de ahorro y crédito y servicios y por agrupaciones de trabajadores (c) Establecimientos públicos de educación superior y (d) equipo de asistencia médica y quirúrgico, medicamentos y materias primas para su fabricación en Costa Rica.

7 El sistema armonizado de designación y codificación de mercancías (SA) se refiere al sistema creado por la Organización Mundial de Aduanas que agrupa todos bienes de forma sistemática de acuerdo con criterios tales como origen (animal, vegetal, mineral), grado de elaboración, usos y aplicaciones. 
los aranceles consolidados se refieren al nivel del arancel que el país se compromete a mantener. Los aranceles consolidados son diferentes a los aranceles aplicados. Los aranceles aplicados se refieren al derecho arancelario que efectivamente se aplica en el momento de la importación de las mercancías. En otras palabras, el arancel aplicado puede ser igual o inferior al arancel consolidado, pero no superior.

Por otro lado, Costa Rica tiene un arancel externo común (AEC) armonizado a un $95,7 \%$ con los demás países que conforman el Mercado Común Centroamericano (MCCA) (SIECA, 2009), de los cuales, un 6,46\% de ese $4,3 \%$ de arancel no armonizado representan productos agrícolas. El AEC se refiere a un sistema en donde los miembros de una unión aduanera definen un mismo arancel aplicado para todo el universo de mercancías. En el caso de Centroamérica, este AEC está identificado sobre la base de 8 dígitos -los 6 dígitos del SA y dos dígitos adicionales para identificar el AEC-.

La definición del AEC de Centroamérica sigue una política arancelaria común. Esta política arancelaria está establecida en la Resolución N.o 26-96 (COMRIEDRE-IV) con fecha del 12 de diciembre del 1995. En dicha resolución se indican 4 niveles arancelarios: (i) $0 \%$ aplicado a aquellas materias primas que no se elaboran en los países centroamericanos, (ii) $5 \%$ aplicado a materias primas que son elaborados en los países centroamericanos, (iii) $10 \%$ a materiales intermedios y bienes de capital producidos en Centroamérica y (iv) $15 \%$ a bienes finales.

La política arancelaria indicada anteriormente fue creada para incentivar la producción de bienes finales a lo interno de Centroamérica. El espíritu de esta resolución era desmotivar la importación de mercancías finales para que estas fueran manufacturadas con materias primas competitivas.

El AEC no está armonizado al $100 \%$. Para todos aquellos aranceles que no están armonizados, cada país centroamericano -de forma individual- tiene la potestad de modificar el arancel aplicado, siempre y cuando no exceda el arancel consolidado ante la OMC. Sin embargo, para modificar el arancel aplicado incluido en el AEC, la decisión debe ser tomada en consenso entre los países miembros del MCCA (Resolución N.o 26-96 del COMRIEDRE-IV). 


\section{Arancel NMF consolidado por Costa Rica ante la OMC}

Costa Rica ha consolidado ante la OMC el 100\% de las líneas arancelarias. La tabla 1 presenta un resumen sobre los aranceles consolidados por Costa Rica en su Lista de concesiones de la OMC.

\section{Tabla 1}

Resumen sobre los aranceles consolidados

Sistema Arancelario del 2002

Costa Rica

\begin{tabular}{lc}
\hline Criterio & Dato \\
Cantidad de líneas arancelarias a nivel de 6 dígitos & 5224 \\
Cantidad de líneas arancelarias a nivel de 8 dígitos & 5940 \\
Cantidad de líneas arancelarias consolidadas, en porcentaje & $100 \%$ \\
Cantidad de líneas con derechos ad valorem, en porcentaje & $100 \%$ \\
Arancel aplicado máximo, en porcentaje & $233.1 \%$ \\
Arancel aplicado mínimo, en porcentaje & $0 \%$ \\
Promedio total del arancel ad valorem, en porcentaje & $43.1 \%$ \\
Promedio del arancel ad valorem para productos agropecuarios, & $43.2 \%$ \\
en porcentaje & \\
Promedio del arancel ad valorem para productos no agropecuarios, & $43.1 \%$ \\
en porcentaje & $3.80 \%$ \\
Cantidad de líneas libres de derecho a 8 dígitos, en porcentaje & \\
\hline
\end{tabular}

Nota: Elaboración propia, con base en la información extraída de la Función de Descarga de Aranceles de la OMC $\left(2015^{\mathrm{a}}\right)$.

Como se observa en la tabla 1, la totalidad de las líneas arancelarias del SA tiene un arancel ad valorem, es decir, el cálculo del arancel se realiza con base en un porcentaje aplicado al valor de las mercancías. Es importante que la totalidad de los aranceles consolidados estén en ad valorem, ya que su aplicación, así como el proceso de recorte, es más sencillo que los otros métodos descritos.

La tabla 1 también revela que únicamente 231 líneas arancelarias o un 3.8\% de las líneas posee un arancel consolidado de $0 \%$. Esto quiere decir que estos productos no podrán utilizar un arancel aplicado superior al $0 \%$-salvo casos justificados y establecidos ante la OMC-. Entre los productos que se encuentran con arancel consolidado $0 \%$ están: aparatos de computación, productos ópticos entre otros. Esto refleja que estos productos descritos no presentan ninguna 
sensibilidad en el mercado costarricense, además, algunos de ellos forman parte de las mercancías que figuran en el Acuerdo de la OMC sobre Tecnología de la Información y, por ende, debe aplicar un $0 \%$ arancel.

El arancel consolidado promedio es de un $43 \%$ aproximadamente, sin embargo, el nivel arancelario depende mucho del tipo de producto. En general, la OMC define dos grandes sectores, el "agropecuario" y el "no agropecuario". El Anexo 1 del Acuerdo sobre Agricultura de la OMC define el sector agropecuario como todos aquellos productos clasificados desde el capítulo 1 al 24, excluyendo capítulo 3 e incluyendo otros productos ${ }^{8}$. En la tabla 1, se observa que, en realidad, a nivel de arancel consolidado, no hay una verdadera distinción en el promedio arancelario consolidado de los productos agropecuarios y no agropecuarios, sin embargo, esto no significa que no haya diferencias a nivel de arancel aplicado. Esta información se presentará en un apartado posterior.

Para lograr identificar de manera más efectiva los niveles arancelarios consolidados de los productos, la tabla 2 muestra para cada capítulo del SA, el promedio consolidado, el porcentaje de líneas arancelarias, así como el arancel máximo y mínimo consolidado. Esta información es importante, ya que ayuda a identificar si las mercancías son o no consideradas como sensibles en el país.

\section{Tabla 2}

Aranceles consolidados por capítulo del SA 2002 Costa Rica

\begin{tabular}{lcccc}
\multicolumn{5}{c}{ Derechos consolidados } \\
Capítulos & Promedio & Exento en \% & Arancel máximo & Arancel mínimo \\
\hline Capítulo 1 & 35.65 & 0 & 45 & 10 \\
Capítulo 2 & 56.91 & 0 & 233.1 & 20 \\
Capítulo 3 & 45.00 & 0 & 45 & 45 \\
Capítulo 4 & 74.16 & 0 & 94.7 & 5 \\
Capítulo 5 & 45.00 & 0 & 45 & 45 \\
Capítulo 6 & 34.58 & 0 & 45 & 20 \\
Capítulo 7 & 43.61 & 0 & 45 & 5
\end{tabular}

8 Manitol (2905.43), Sorbitol (2905.44), aceites esenciales (33.01), materias albuminoideas, productos a base de almidón o de fécula modificados, colas (35.01 a 35.05), aprestos y productos de acabado (3809.10), sorbitol n.e.p (3823.60), cueros y pieles (41.01 a 41.03), peletería en bruto (43.01), seda cruda y desperdicios de seda (50.01 a 50.03), lana y pelo (51.01 a 51.03), algodón en rama, desperdicios de algodón y algodón cardado o peinado (52.01 a 52.03), lino en bruto (53.01) y cáñamo en bruto (53.02). 


\begin{tabular}{|c|c|c|c|c|}
\hline \multirow[b]{2}{*}{ Capítulos } & \multicolumn{4}{|c|}{ Derechos consolidados } \\
\hline & Promedio & Exento en \% & Arancel máximo & Arancel mínimo \\
\hline Capítulo 8 & 42.86 & 0 & 45 & 20 \\
\hline Capítulo 9 & 43.13 & 0 & 45 & 10 \\
\hline Capítulo 10 & 33.97 & 0 & 45 & 1 \\
\hline Capítulo 11 & 38.45 & 0 & 45 & 20 \\
\hline Capítulo 12 & 4.64 & 0 & 45 & 1 \\
\hline Capítulo 13 & 45.00 & 0 & 45 & 45 \\
\hline Capítulo 14 & 45.00 & 0 & 45 & 45 \\
\hline Capítulo 15 & 40.54 & 0 & 45 & 5 \\
\hline Capítulo 16 & 68.30 & 0 & 233.1 & 45 \\
\hline Capítulo 17 & 45.00 & 0 & 45 & 45 \\
\hline Capítulo 18 & 47.27 & 0 & 50 & 45 \\
\hline Capítulo 19 & 43.07 & 0 & 45 & 5 \\
\hline Capítulo 20 & 43.17 & 0 & 50 & 5 \\
\hline Capítulo 21 & 47.71 & 0 & 94.7 & 25 \\
\hline Capítulo 22 & 34.83 & 0 & 60 & 40 \\
\hline Capítulo 23 & 40.29 & 0 & 45 & 5 \\
\hline Capítulo 24 & 41.67 & 0 & 45 & 40 \\
\hline Capítulo 25 & 45.00 & 0 & 45 & 45 \\
\hline Capítulo 26 & 45.00 & 0 & 45 & 45 \\
\hline Capítulo 27 & 44.83 & 0 & 45 & 30 \\
\hline Capítulo 28 & 45.00 & 0 & 45 & 45 \\
\hline Capítulo 29 & 44.71 & 0 & 45 & 20 \\
\hline Capítulo 30 & 44.67 & 0 & 45 & 40 \\
\hline Capítulo 31 & 43.08 & 0 & 45 & 20 \\
\hline Capítulo 32 & 42.97 & 0 & 45 & 25 \\
\hline Capítulo 33 & 45.00 & 0 & 45 & 45 \\
\hline Capítulo 34 & 45.00 & 0 & 45 & 45 \\
\hline Capítulo 35 & 38.33 & 0 & 45 & 20 \\
\hline Capítulo 36 & 45.00 & 0 & 45 & 45 \\
\hline Capítulo 37 & 45.00 & 0 & 45 & 45 \\
\hline Capítulo 38 & 44.05 & 1.39 & 45 & 0 \\
\hline Capítulo 39 & 38.43 & 0 & 45 & 5 \\
\hline Capítulo 40 & 45.44 & 0 & 45 & 45 \\
\hline Capítulo 41 & 37.37 & 0 & 45 & 5 \\
\hline
\end{tabular}




\begin{tabular}{|c|c|c|c|c|}
\hline \multirow[b]{2}{*}{ Capítulos } & \multicolumn{4}{|c|}{ Derechos consolidados } \\
\hline & Promedio & Exento en \% & Arancel máximo & Arancel mínimo \\
\hline Capítulo 42 & 42.27 & 0 & 45 & 40 \\
\hline Capítulo 43 & 47.86 & 0 & 60 & 45 \\
\hline Capítulo 44 & 37.92 & 0 & 45 & 10 \\
\hline Capítulo 45 & 45.00 & 0 & 45 & 45 \\
\hline Capítulo 46 & 45.00 & 0 & 45 & 45 \\
\hline Capítulo 47 & 40.00 & 0 & 45 & 20 \\
\hline Capítulo 48 & 46.16 & 0 & 50 & 30 \\
\hline Capítulo 49 & 46.58 & 0 & 55 & 45 \\
\hline Capítulo 50 & 45.00 & 0 & 45 & 45 \\
\hline Capítulo 51 & 45.00 & 0 & 45 & 45 \\
\hline Capítulo 52 & 45.00 & 0 & 45 & 45 \\
\hline Capítulo 53 & 45.00 & 0 & 45 & 45 \\
\hline Capítulo 54 & 45.00 & 0 & 45 & 45 \\
\hline Capítulo 55 & 45.00 & 0 & 45 & 45 \\
\hline Capítulo 56 & 45.12 & 0 & 60 & 45 \\
\hline Capítulo 57 & 45.00 & 0 & 45 & 45 \\
\hline Capítulo 58 & 45.37 & 0 & 60 & 45 \\
\hline Capítulo 59 & 45.00 & 0 & 45 & 45 \\
\hline Capítulo 60 & 45.00 & 0 & 45 & 45 \\
\hline Capítulo 61 & 45.00 & 0 & 45 & 45 \\
\hline Capítulo 62 & 45.00 & 0 & 45 & 45 \\
\hline Capítulo 63 & 45.00 & 0 & 45 & 45 \\
\hline Capítulo 64 & 54.83 & 0 & 79 & 45 \\
\hline Capítulo 65 & 45.00 & 0 & 45 & 45 \\
\hline Capítulo 66 & 45.00 & 0 & 45 & 45 \\
\hline Capítulo 67 & 45.00 & 0 & 45 & 45 \\
\hline Capítulo 68 & 44.69 & 0 & 45 & 45 \\
\hline Capítulo 69 & 46.90 & 0 & 60 & 45 \\
\hline Capítulo 70 & 43.29 & 0 & 45 & 25 \\
\hline Capítulo 71 & 44.72 & 0 & 45 & 40 \\
\hline Capítulo 72 & 35.50 & 0 & 45 & 20 \\
\hline Capítulo 73 & 43.39 & 0 & 45 & 45 \\
\hline Capítulo 74 & 45.00 & 0 & 45 & 45 \\
\hline Capítulo 75 & 45.00 & 0 & 45 & 45 \\
\hline
\end{tabular}




\begin{tabular}{lcccc} 
& \multicolumn{4}{c}{ Derechos consolidados } \\
Capítulos & Promedio & Exento en \% & Arancel máximo & Arancel mínimo \\
\hline Capítulo 76 & 45.00 & 0 & 45 & 45 \\
Capítulo 78 & 45.00 & 0 & 45 & 45 \\
Capítulo 79 & 45.00 & 0 & 45 & 45 \\
Capítulo 80 & 41.88 & 0 & 45 & 20 \\
Capítulo 81 & 45.00 & 0 & 45 & 45 \\
Capítulo 82 & 45.00 & 0 & 45 & 45 \\
Capítulo 83 & 45.00 & 0 & 45 & 45 \\
Capítulo 84 & 40.97 & 11.15 & 45 & 0 \\
Capítulo 85 & 34.04 & 30.66 & 45 & 0 \\
Capítulo 86 & 45.00 & 0 & 45 & 45 \\
Capítulo 87 & 55.43 & 0 & 100 & 20 \\
Capítulo 88 & 45.00 & 0 & 45 & 45 \\
Capítulo 89 & 45.00 & 0 & 45 & 45 \\
Capítulo 90 & 35.86 & 21.31 & 45 & 0 \\
Capítulo 91 & 45.00 & 0 & 45 & 45 \\
Capítulo 92 & 45.00 & 0 & 45 & 45 \\
Capítulo 93 & 45.00 & 0 & 45 & 45 \\
Capítulo 94 & 41.55 & 0 & 45 & 40 \\
Capítulo 95 & 42.73 & 0 & 45 & 45 \\
Capítulo 96 & 44.77 & 0 & 45 & 45 \\
Capítulo 97 & 44.75 & 0 & & 45 \\
\hline
\end{tabular}

Nota: Elaboración propia, con base en la información extraída de la Función de Descarga de Aranceles de la OMC, 2015a.

En la tabla 2, se puede observar que hay mercancías que son tradicionalmente sensibles como indican las tendencias mundiales. En el caso del análisis de los aranceles consolidados, se puede observar que el Capítulo 4 del SA (productos lácteos) posee el arancel promedio consolidado más elevado y que el Capítulo 2 (carnes y despojos comestibles) y Capítulo 16 (alimentos procesados) poseen los aranceles consolidados más elevados (233\%). Dicha evidencia, en el caso de Costa Rica, coindice con las tendencias mundiales ya que, según esta tónica, por lo general los productos agropecuarios son propensos a tener aranceles superiores a los aranceles de los productos no agropecuarios. La tabla 3 muestra la distribución de los aranceles según las frecuencias arancelarias. 
REVISTA 90.1

Tabla 3

Distribución de los Aranceles Consolidados

Productos agropecuarios y no agropecuarios

Costa Rica

Porcentaje

\begin{tabular}{ccc}
$\begin{array}{c}\text { Distribución } \\
\text { arancelaria }\end{array}$ & $\begin{array}{c}\text { Productos agropecuarios } \\
\text { \% líneas arancelarias }\end{array}$ & $\begin{array}{c}\text { Productos no agropecuarios } \\
\text { \% líneas arancelarias }\end{array}$ \\
\hline Exentas & 0 & 2.3 \\
$\mathbf{0} \leq \mathbf{5}$ & 6.1 & 0.4 \\
$\mathbf{5} \leq \mathbf{1 0}$ & 2.0 & 0.3 \\
$\mathbf{1 0 \leq 1 5}$ & 1.2 & 0.1 \\
$\mathbf{1 5} \leq \mathbf{2 5}$ & 4.2 & 3.5 \\
$\mathbf{2 5} \leq \mathbf{5 0}$ & 82.5 & 92.4 \\
$\mathbf{5 0 \leq 1 0 0}$ & 3.0 & 1.0 \\
$>\mathbf{1 0 0}$ & 1.0 & 0 \\
No ad valorem & 0 & 0 \\
\hline
\end{tabular}

Nota: OMC, 2015c.

En la tabla 3, se puede apreciar que 1\% de las líneas arancelarias de los productos agropecuarios tienen un arancel superior al 100\% y un 3\% de las líneas arancelarias con un arancel consolidado igual a 50 e inferior o igual a 100. Esto, a diferencia de los productos no agropecuarios que no tienen ninguna línea arancelaria con arancel superior a $100 \%$ y únicamente $1 \%$ en líneas entre 50 y 100 por ciento. Lo anterior quiere decir que productos agropecuarios tienden a tener un arancel superior a los no agropecuarios.

Para tener mayor claridad en cuanto a la distribución arancelaria consolidada, en la tabla 4 se determina el promedio del arancel consolidado según diferentes sectores, el porcentaje de estos que están exentos del pago de aranceles así como el tope máximo aplicable a ese sector. Esta información es de gran relevancia, ya que permite definir de manera más exacta aquellos sectores considerados como sensibles en Costa Rica. 


\section{Tabla 4}

Promedio de Arancel Consolidado

por grupos de productos, dividido en bienes agropecuarios y no agropecuarios

Costa Rica

\begin{tabular}{|c|c|c|c|}
\hline \multirow[t]{2}{*}{ Sectores } & \multicolumn{3}{|c|}{ Derechos consolidados } \\
\hline & Promedio & $\begin{array}{c}\text { Exentos } \\
(\text { en } \%)\end{array}$ & Máximo \\
\hline Productos animales & 56.0 & 0 & 233 \\
\hline Productos lácteos & 84.8 & 0 & 95 \\
\hline Frutas, legumbres, plantas & 41.8 & 0 & 45 \\
\hline Café y té & 46.0 & 0 & 50 \\
\hline Cereales y otras preparaciones & 40.3 & 0 & 95 \\
\hline Semillas oleaginosas, grasas y aceites & 28.7 & 0 & 45 \\
\hline Azúcares y artículos de confitería & 45.0 & 0 & 45 \\
\hline Bebidas y tabaco & 45.4 & 0 & 60 \\
\hline Algodón & 45.0 & 0 & 45 \\
\hline Otros productos agrícolas & 37.7 & 0 & 45 \\
\hline Pescados y sus productos & 46.0 & 0 & 60 \\
\hline Metales y minerales & 43.0 & 0.1 & 60 \\
\hline Petróleo & 43.5 & 0 & 45 \\
\hline Productos químicos & 43.6 & 0.1 & 55 \\
\hline Madera, papel, etc. & 42.3 & 0 & 55 \\
\hline Textiles & 45.1 & 0 & 70 \\
\hline Prendas de vestir & 45.0 & 0 & 45 \\
\hline Cueros, calzados, etc. & 45.1 & 0 & 70 \\
\hline Máquinas no eléctricas & 41.2 & 5.7 & 50 \\
\hline Máquinas eléctricas & 35.6 & 19.4 & 45 \\
\hline Material de transporte & 51.5 & 0 & 100 \\
\hline Manufacturas n.e.p & 41.4 & 5.9 & 45 \\
\hline
\end{tabular}

Nota: Elaboración propia, a partir de la información de Costa Rica y la OMC, 2015b.

La información presentada en la tabla 4 también confirma que los productos agropecuarios son más sensibles que los no agropecuarios. Según los datos, los sectores más sensibles son los productos animales y los productos lácteos con 
aranceles hasta $233 \%$ y $95 \%$ respectivamente. A la vez, en la tabla 4 se puede observar que la maquinaria eléctrica es uno de los sectores en donde existe la mayor cantidad de mercancías exentas del pago de arancel. Esto refuerza la idea de que estos tipos de productos no son sensibles para el país y, además, son productos que se encuentran incluidos dentro del Acuerdo de la OMC sobre Tecnología de la Información -por lo tanto tienen $0 \%$ arancel-.

En la tabla 5, se verán los 10 principales productos con los aranceles consolidados más altos de Costa Rica. Dicho cuadro muestra que, de los diez productos, seis son productos agrícolas y uno corresponde a textiles y calzado. Esto demuestra que Costa Rica persigue las tendencias mundiales respecto a los tipos de mercancías con los mayores aranceles. En un apartado posterior se observa que los principales productos con el arancel consolidado más elevado, resultan ser análogos a los principales productos con el arancel aplicado, a excepción de tres productos.

\section{Tabla 5}

Principales 10 productos con el Arancel Consolidado más elevado Costa Rica

\begin{tabular}{|c|c|c|c|}
\hline Posición & Descripción & $\begin{array}{c}\text { Partida } \\
\text { arancelaria }\end{array}$ & $\begin{array}{c}\text { Arancel } \\
\%\end{array}$ \\
\hline 1 & $\begin{array}{l}\text { Trozos y despojos comestibles de gallo, gallina, } \\
\text { pavo o pato }\end{array}$ & 207 & $233.1 \%$ \\
\hline 2 & $\begin{array}{l}\text { Embutidos de carne y otras preparaciones de } \\
\text { carne y conservas de carne }\end{array}$ & 1601 y 1602 & $233.1 \%$ \\
\hline 3 & $\begin{array}{l}\text { Vehículos, automóviles de turismo, vehículos } \\
\text { para transporte de mercancías, chasis de trac- } \\
\text { tores, carrocerías de automóviles de turismo, } \\
\text { carrocerías de tractores }\end{array}$ & $\begin{array}{l}8703,8704 \\
8706,8707\end{array}$ & $100 \%$ \\
\hline 4 & $\begin{array}{l}\text { Leche y nata "crema", yogur, suero de mante- } \\
\text { quilla, lactosuero, productos constituidos por } \\
\text { los componentes naturales de la leche, man- } \\
\text { tequilla de manteca, grasas de la leche, queso } \\
\text { fresco "sin madurar", queso fundido }\end{array}$ & $\begin{array}{l}\text { 0401, } 0402, \\
0403,0404, \\
0405,0406\end{array}$ & $94.7 \%$ \\
\hline 5 & Helados & 2105 & $94.7 \%$ \\
\hline 6 & Calzados y sus partes & $\begin{array}{l}6403,6405 \\
6406\end{array}$ & $70 \%$ \\
\hline
\end{tabular}




\begin{tabular}{clcc}
\hline Posición & Descripción & $\begin{array}{c}\text { Partida } \\
\text { arancelaria }\end{array}$ & $\begin{array}{c}\text { Arancel } \\
\%\end{array}$ \\
7 & $\begin{array}{l}\text { Preparaciones y conservas de salmón, anchoas, } \\
\text { cangrejo, camarón, bogavante, crustáceos, mo- } \\
\text { luscos, caviar }\end{array}$ & 1604,1605 & $60 \%$ \\
8 & $\begin{array}{l}\text { Vermut y demás vinos de uvas, sidra } \\
9\end{array}$ & $\begin{array}{l}\text { Mezclas de alquivencenos y mezclas de alqui- } \\
\text { naftalenos }\end{array}$ & 2205,2206 \\
10 & Libros, folletos, diccionarios & $60 \%$ & $55 \%$ \\
\hline
\end{tabular}

Nota: Elaboración propia, a partir de la información de Costa Rica y la OMC (2015b).

\section{Arancel aplicado en Costa Rica}

Como se indicó anteriormente, el arancel aplicado es diferente al arancel consolidado. El arancel aplicado es el efectivamente recolectado en el momento de la importación de las mercancías. Por lo general, los aranceles aplicados son inferiores a los aranceles consolidados; pero no pueden ser superiores al consolidado. La tabla 6 muestra un resumen sobre los aranceles aplicados de Costa Rica.

\section{Tabla 6}

Resumen sobre los Aranceles Aplicados

Sistema Arancelario del 2012

Costa Rica

\section{Criterio}

Dato

Cantidad de líneas arancelarias a nivel de 6 dígitos

5205

Cantidad de líneas arancelarias a nivel de 8 dígitos

10302

Cantidad de líneas con derechos ad valorem, en porcentaje

$100 \%$

Arancel aplicado máximo, en porcentaje

$150 \%$

Arancel aplicado mínimo, en porcentaje

$0 \%$

Promedio total del arancel ad valorem, en porcentaje

$5.57 \%$

Promedio del arancel ad valorem para productos agropecuarios, en porcentaje 
REVISTA 90.1

Promedio del arancel ad valorem para productos no agropecuarios, en porcentaje

Nota: Elaboración propia, con base en la información extraída de la Función de Descarga de Aranceles de la OMC (2015a).

Analizando la información presentada en tabla 6, se puede observar que efectivamente el arancel promedio aplicado es $87 \%$ menos que el arancel consolidado (el arancel consolidado promedio ronda un $43.1 \%$-según tabla 1- mientras el aplicado es un 5.57\%). Adicionalmente, se observa que casi un 50\% de las líneas arancelares tienen ya un $0 \%$ de arancel aplicado. Esto quiere decir que casi un $50 \%$ de las líneas arancelarias no tendrán un beneficio real en los TLC, ya que el arancel NMF es igual a $0 \%$. Otro aspecto importante que hay que destacar en la tabla 6 es que, al igual que en la tabla 1, los productos agropecuarios siguen teniendo aranceles superiores a los productos no agropecuarios.

Con el fin de determinar, de manera más efectiva, el aprovechamiento potencial que un TLC puede tener con respecto al término de los aranceles, la tabla 7 muestra la distribución de los aranceles aplicados, según productos agrícolas y no agrícolas en Costa Rica.

\section{Tabla 7}

Distribución de aranceles aplicados

Productos agropecuarios y no agropecuarios

Costa Rica

Porcentaje

\begin{tabular}{ccc}
$\begin{array}{c}\text { Distribución } \\
\text { arancelaria }\end{array}$ & $\begin{array}{c}\text { Productos agropecuarios } \\
\text { \% líneas arancelarias }\end{array}$ & $\begin{array}{c}\text { Productos no agropecuarios } \\
\text { \% líneas arancelarias }\end{array}$ \\
\hline $\begin{array}{c}\text { Exentas } \\
\mathbf{0} \leq \mathbf{5}\end{array}$ & 30.1 & 54.9 \\
$\mathbf{5} \leq \mathbf{1 0}$ & 10.5 & 10.6 \\
$\mathbf{1 0} \leq \mathbf{1 5}$ & 9.8 & 14.9 \\
$\mathbf{1 5} \leq \mathbf{2 5}$ & 41 & 19.6 \\
$\mathbf{2 5} \leq \mathbf{5 0}$ & 0.2 & 0 \\
$\mathbf{5 0} \leq \mathbf{1 0 0}$ & 6.8 & 0 \\
\end{tabular}




\begin{tabular}{ccc}
$\begin{array}{c}\text { Distribución } \\
\text { arancelaria }\end{array}$ & $\begin{array}{c}\text { Productos agropecuarios } \\
\text { \% líneas arancelarias }\end{array}$ & $\begin{array}{c}\text { Productos no agropecuarios } \\
\text { \% líneas arancelarias }\end{array}$ \\
\hline >100 & 0.3 & 0 \\
No ad valorem & 0 & 0 \\
\cline { 2 - 3 }
\end{tabular}

Nota: Elaboración propia, a partir de la información de Costa Rica y la OMC, 2015 b.

La tabla 7 revela que los productos no agropecuarios son más propensos a no pagar arancel, es decir, que el arancel aplicado es de $0 \%$. A la vez, se muestra que el arancel máximo de los productos no agropecuarios es un $15 \%$, mientras que los productos agropecuarios tienen aproximadamente $9 \%$ de líneas arancelarias con un arancel superior al 15\%. Lo anterior reafirma que los productos agropecuarios son más propensos a ser sensibles y, por ende, sus aranceles son superiores.

A la vez, la tabla 7 muestra que más de un 10\% de las líneas arancelarias de productos agropecuarios y de productos no agropecuarios tienen un margen arancelario entre un $0 \%$ y 5\%. Según las tendencias desarrolladas en el Informe sobre el Comercio Mundial (OMC, 2011b, p. 81), cuando los beneficios arancelarios son reducidos, las empresas prefieren no aplicar el TLC, ya que deben incurrir en gastos para el cumplimiento de las reglas de origen cuando a la larga no son suficientes para cubrir los gastos iniciales. Asumiendo que la tendencia se cumple en Costa Rica, las empresas no utilizan la preferencia arancelaria en más de un $21 \%$ de las líneas arancelarias de Costa Rica $-10.5 \%$ de las líneas arancelarias de productos agrícolas y $10.6 \%$ de líneas arancelarias de productos no agrícolas-.

\section{Tabla 8}

Promedio de arancel aplicado

por grupos de productos

Costa Rica

\begin{tabular}{|c|c|c|c|}
\hline \multirow[t]{2}{*}{ Sectores } & \multicolumn{3}{|c|}{ Derechos aplicados } \\
\hline & Promedio & $\begin{array}{c}\text { Exentos en } \\
\%\end{array}$ & Máximo \\
\hline Productos animales & 20.0 & 14 & 150 \\
\hline Productos lácteos & 43.7 & 6 & 65 \\
\hline Frutas, legumbres, plantas & 11.5 & 20 & 45 \\
\hline Café y té & 12.2 & 6 & 14 \\
\hline
\end{tabular}


REVISTA90.1

\begin{tabular}{|c|c|c|c|}
\hline \multirow[t]{2}{*}{ Sectores } & \multicolumn{3}{|c|}{ Derechos aplicados } \\
\hline & Promedio & $\begin{array}{c}\text { Exentos en } \\
\%\end{array}$ & Máximo \\
\hline Cereales y otras preparaciones & 8.3 & 43 & 65 \\
\hline $\begin{array}{l}\text { Semillas oleaginosas, grasas } \\
\text { y aceites }\end{array}$ & 5.5 & 36 & 14 \\
\hline Azúcares y artículos de confitería & 18.9 & 23 & 45 \\
\hline Bebidas y tabaco & 12.8 & 7 & 40 \\
\hline Algodón & 0.0 & 100 & 0 \\
\hline Otros productos agrícolas & 3.1 & 60 & 14 \\
\hline Pescados y sus productos & 10.7 & 11 & 45 \\
\hline Metales y minerales & 3.0 & 66.7 & 14 \\
\hline Petróleo & 6.8 & 33 & 14 \\
\hline Productos químicos & 1.8 & 79.9 & 14 \\
\hline Madera, papel, etc. & 5.9 & 45 & 14 \\
\hline Textiles & 7.5 & 14 & 14 \\
\hline Prendas de vestir & 13.9 & 1 & 14 \\
\hline Cueros, calzados, etc. & 7.3 & 23 & 14 \\
\hline Máquinas no eléctricas & 1.1 & 90.1 & 14 \\
\hline Máquinas eléctricas & 2.1 & 79.8 & 14 \\
\hline Material de transporte & 3.9 & 62 & 14 \\
\hline Manufacturas n.e.p & 6.1 & 44.5 & 14 \\
\hline
\end{tabular}

Nota: OMC, 2015c.

Según la información presentada en la tabla 8 , se nota que a pesar de que el promedio del arancel consolidado para los productos no agropecuarios es de un $43.1 \%$-según la tabla 1-, el arancel aplicado para todos estos productos -excepto pescado y sus subproductos- aplican un arancel máximo de 14\% (un 15\% si se suma la Ley No. 6946). Esto no sucede con respecto a los productos agropecuarios; de los 10 sectores de los productos agropecuarios dos de ellos tienen el arancel aplicado máximo igual que arancel máximo consolidado, cuatro sectores tuvieron en el arancel aplicado solo un 35\% de reducción con respecto al arancel consolidado. Se confirma, así, que los productos agropecuarios son más sensibles que los no agropecuarios. 


\section{Tabla 9}

Principales 10 productos con el Arancel Aplicado más alto Costa Rica

\begin{tabular}{|c|c|c|c|}
\hline Posición & Descripción & $\begin{array}{c}\text { Partida } \\
\text { arancelaria }\end{array}$ & $\begin{array}{l}\text { Arancel } \\
\text { aplicado }\end{array}$ \\
\hline 1 & $\begin{array}{l}\text { Trozos y despojos comestibles de gallo, gallina, } \\
\text { pavo o pato }\end{array}$ & 207 & 150 \\
\hline 2 & $\begin{array}{l}\text { Embutidos de carne y otras preparaciones de } \\
\text { carne y conservas de carne }\end{array}$ & 1601 y 1602 & 150 \\
\hline 3 & $\begin{array}{l}\text { Leche y nata "crema", yogur, suero de mante- } \\
\text { quilla, lactosuero, productos constituidos por } \\
\text { los componentes naturales de la leche, man- } \\
\text { tequilla de manteca, grasas de la leche, queso } \\
\text { fresco "sin madurar", queso fundido }\end{array}$ & $\begin{array}{c}0401,0402 \\
0403,0405 \\
0406\end{array}$ & 65 \\
\hline 4 & Helados & 2105 & 65 \\
\hline 5 & Queso & 406 & 50 \\
\hline 6 & Carne de cerdo y sus despojos; jamones, tocino & $\begin{array}{l}0203,0206 \\
0210\end{array}$ & 45 \\
\hline 7 & Filetes congelados de bagre & 304 & 45 \\
\hline 8 & Papas & 0701,0710 & 45 \\
\hline 9 & Cebollas & 703 & 45 \\
\hline 10 & Azúcar de caña y de remolacha & 1701 & 45 \\
\hline
\end{tabular}

Nota: Elaboración propia, a partir de la información de Costa Rica y la OMC, 2015b.

Si se analiza la tabla 9 y se compara con la 5 , referente a los principales 10 productos con el arancel consolidado más alto, se determina que los primeros dos productos coinciden al $100 \%$. En cuanto al arancel aplicado, los vehículos, los calzados, el vermut y vinos, las mezclas de alquibencenos y alquinaftalenos y los libros, folletos y diccionarios no se encuentran entre los principales productos con aranceles aplicados más altos.

A su vez, la tabla 9 revela que la totalidad de los 10 principales productos de importación con arancel superior al $45 \%$ en Costa Rica corresponden a productos agropecuarios, según la definición del Acuerdo sobre Agricultura de la OMC. Esto sigue confirmando que los productos agropecuarios son más sensibles en Costa Rica que los productos no agropecuarios. 
Tabla 10

Arancel Aplicado de los principales 25 productos de importación en el 2014 Costa Rica

\begin{tabular}{|c|c|c|c|c|}
\hline $\mathbf{N}^{0}$ & Subpartida & $\begin{array}{c}\text { Participación } \\
\%\end{array}$ & $\begin{array}{c}\text { DAI } \\
\%\end{array}$ & $\begin{array}{c}\text { Ley No. } 6946 \\
\%\end{array}$ \\
\hline 1 & 854231 & $8,40 \%$ & 0 & 1 \\
\hline 2 & 271019 & $6,80 \%$ & 0 & 1 \\
\hline 3 & 271012 & $5,50 \%$ & 0 & 1 \\
\hline 4 & 853400 & $3,80 \%$ & 0 & 0 \\
\hline 5 & 851712 & $2,10 \%$ & 0 & 1 \\
\hline 6 & 300490 & $1,90 \%$ & 0 & 1 \\
\hline 7 & 870323 & $1,40 \%$ & 14 & 1 \\
\hline 8 & 100590 & $1,10 \%$ & 0 & 1 \\
\hline 9 & 860900 & $1,00 \%$ & 0 & 1 \\
\hline 10 & 392690 & $0,90 \%$ & 5 & 1 \\
\hline 11 & 120190 & $0,90 \%$ & 0 & 1 \\
\hline 12 & 740811 & $0,80 \%$ & 0 & 1 \\
\hline 13 & 847130 & $0,70 \%$ & 0 & 0 \\
\hline 14 & 210690 & $0,70 \%$ & 0 & 1 \\
\hline 15 & 480411 & $0,70 \%$ & 0 & 1 \\
\hline 16 & 870421 & $0,60 \%$ & 0 & 1 \\
\hline 17 & 852872 & $0,60 \%$ & 0 & 1 \\
\hline 18 & 732690 & $0,60 \%$ & 0 & 1 \\
\hline 19 & 271111 & $0,50 \%$ & 0 & 1 \\
\hline 20 & 854290 & $0,50 \%$ & 0 & 0 \\
\hline 21 & 901839 & $0,50 \%$ & 0 & 1 \\
\hline 22 & 300210 & $0,40 \%$ & 0 & 1 \\
\hline 23 & 870333 & $0,40 \%$ & 14 & 1 \\
\hline 24 & 870322 & $0,40 \%$ & 14 & 1 \\
\hline 25 & 851770 & $0,40 \%$ & 0 & 0 \\
\hline \multicolumn{2}{|c|}{ SUBTOTAL } & $41,70 \%$ & & \\
\hline \multicolumn{2}{|c|}{ Las demás } & $58,30 \%$ & & \\
\hline \multicolumn{2}{|c|}{ TOTAL } & $100,00 \%$ & & \\
\hline
\end{tabular}

Nota: Elaboración propia, con datos de COMEX, 2015 e información del arancel vigente. 
La tabla 10 muestra los 25 principales productos que Costa Rica importa del resto del mundo y que representan casi el $42 \%$ de las importaciones hacia el país. Estos datos revelan que la mayoría de estos productos no gozan de un alto margen de preferencias arancelarias. Lo anterior se debe a que, de los 25 productos de la tabla 10, hay 21 que no pagan DAI, solamente pagan el $1 \%$ de la Ley N.o 6946 - excepto 4 productos que no pagan ni DAI ni Ley N.o 6946-.

A la vez, la tabla 10 revela que únicamente 2 de esos productos son agropecuarios (maíz y soya), sin embargo, son productos agropecuarios no sensibles, ya que el arancel aplicado es casi nulo -únicamente se le aplica $1 \%$ de la Ley No. 6946-. Las tres subpartidas con el arancel aplicado más elevado pertenecen al sector automovilístico (con un 14\% de DAI más 1\% de Ley N.o 6946). Además de estos productos, algunas manufacturas de plástico son los otros productos que tienen un DAI aplicado de 5\% más 1\% de la Ley N.o 6946.

En ese sentido, es posible creer que, al menos para estos principales productos que importa Costa Rica, la tasa de utilización de preferencias arancelarias es relativamente baja.

\section{Contingentes arancelarios}

Durante las 8 rondas de negociaciones comerciales de la OMC, los países tuvieron que transformar los derechos arancelarios de tipo específico, compuesto y mixto a ad valorem. Este proceso se denominó el proceso de arancelización. La idea principal de este proceso era facilitar la aplicación de los derechos, facilitar los recortes futuros y brindar mejor acceso a mercados. A pesar de haberse realizado este proceso, muchos de los aranceles consolidados y aplicados se mantuvieron elevados, por lo tanto, los miembros de la OMC consideraban que no había verdaderamente un acceso real a los mercados. De esta manera, se crearon los contingentes arancelarios. Un contingente arancelario es una cuota designada por un país en donde las exportaciones realizadas dentro de esa cuota pagan un arancel inferior al arancel aplicado normalmente; una vez que se termine esa cuota, se deberán pagar los aranceles sin trato preferencial. Dichos contingentes pueden establecerse en dos ámbitos: contingentes otorgados dentro de la OMC y los contingentes creados dentro de los TLC.

Ahora bien, no todos los países de la OMC tienen contingentes OMC. De hecho, de los 164 países miembros de la OMC, solo 43 de ellos tienen contingentes OMC. Los productos con contingentes OMC asignados en Costa Rica en el año 
$2014^{9}$ son: trozos o despojos de aves; leche; suero de mantequilla, leche y nata cuajadas, yogur, kéfir y demás leches y natas fermentadas o acidificadas incluso concentradas, con adición de azúcar u otro edulcorante, aromatizados o con frutas o cacao; mantequilla y demás materias grasas de la leche, pastas lácteas para untar; queso fresco incluido el lactosuero y el requesón; queso fundido, excepto el rayado o en polvo; embutidos y productos similares de las aves de la partida 0105; preparaciones y conservas homogeneizadas de carnes y despojos de la partida 0105; las demás preparaciones y conservas de gallo o gallina; y helados, incluso con cacao.

La lista con los productos a los cuales se les aplica contingente OMC corresponde, justamente, con aquellos productos con un arancel aplicado y consolidado alto.

Los contingentes creados dentro de los acuerdos comerciales permiten un mayor acceso a mercados y una liberalización, mediante un trato preferencial, al menos para una cantidad volumétrica. Los productos a los que se les aplican contingentes arancelarios en el marco de un TLC son:

- CAFTA-DR (País de origen: Estados Unidos): papa fresca, cebollas frescas, carne de cerdo, muslos de pollo, leche en polvo, mantequilla, quesos, helados, otros productos lácteos, arroz pilado

- CAFTA-DR (País de origen: República Dominicana): pechugas de pollo, leche en polvo

- TLC Costa Rica-Canadá: carne de animales de la especie porcina, fresca, refrigerada o congelada; despojos comestibles de animales de la especie porcina, frescos, refrigerados o congelados; despojos comestibles de animales de la especie porcina, frescos o refrigerados; miel natural; aceites; azúcar refinada obtenida a partir de azúcar en bruto, de caña (no originaria); harina de trigo; habas (frijoles, porotos) de soya; tortas y residuos de la extracción de soya

- Protocolo Bilateral Costa Rica-Panamá al TLC Centroamérica-Panamá: carne de porcino / carne de porcino (arancel 15\%); leche fluida pasteuri-

9 En la asignación de contingentes intervienen distintos actores gubernamentales como el Ministerio de Comercio Exterior (COMEX) y el Ministerio de Agricultura y Ganadería (MAG); como se aprecia más adelante, todas las mercancías bajo contingente son del sector agropecuario. Cada año se dan a conocer los volúmenes disponibles para la asignación de contingentes y la normativa aplicable para el proceso (Reglamento General sobre la Distribución Asignación de Contingentes Arancelarios de Importación y Decreto N. ${ }^{\circ} 36025$-COMEX), a fin de que los importadores interesados puedan aplicar para beneficiarse. 
zada, refrigerada; leche fluida ultrapasteurizada de larga vida (en envases asépticos); leche en polvo, descremada; leche en polvo, entera, a granel (no para reempaque); aceite de palma en bruto y refinado; preparaciones y conservas de carne o despojos, de gallo o gallina; salsas de tomate

- TLC Costa Rica-Perú: carne de bovino

- CAFTA-DR (País de origen: Estados Unidos)- contingente por cosecha-: arroz en granza

- TLC Costa Rica-China: frijol negro (COMEX,2014)

La lógica que se observa en este punto, según la información anterior, es que, efectivamente, las mercancías que conservan contingentes son todos bienes agropecuarios. Esa característica refuerza el argumento de las mercancías sensibles, que se desarrolla a continuación.

\section{Mercancías sensibles}

Costa Rica no tiene criterios definidos para determinar si una mercancía es sensible o no. Sin embargo, hay ciertos elementos que se pueden tomar en consideración para poder identificar estas mercancías. A continuación, se enumeran algunos de estos elementos:

- Mercancías con arancel consolidado y aplicado elevado. Según el GATT, los miembros de la OMC no pueden prohibir la importación o la exportación de mercancías a los Estados miembros salvo algunas excepciones generales (Artículo XX del GATT) o específicas establecidas en cada Tratado. Además, el GATT indica que los miembros no pueden establecer barreras diferentes a las arancelarias para reducir la cantidad de mercancías importadas al territorio. Por lo tanto, cuando una mercancía tiene un arancel elevado, quiere decir que de alguna manera se desea proteger ese sector -independientemente del motivo-. Por lo tanto, mercancías con aranceles elevados son consideradas como sensibles en el país.

- Mercancías que tienen contingentes. Tal y como se mencionó, después del proceso de arancelización, algunos productos mantuvieron un arancel elevado, por ende, la OMC, con el fin de asegurar un acceso a mercado real, decide crear esta figura. Por esta razón, mercancías con contingentes es un buen criterio para catalogarlos como sensibles. 
- Granos básicos (seguridad alimentaria). Por lo general, los gobiernos se preocupan por abastecer a su población de los granos básicos de la canasta básica. En algunas países se cultivan estos granos, aunque ellos no sean eficientes en comparación con el resto del mundo. Sin embargo, los gobiernos tienden a apoyarlos y a protegerlos mediante diferentes mecanismos como aranceles y contingentes para que continúen su producción y no afecten al país en un futuro, en caso de algún desabastecimiento mundial.

- Mercancías producidas dentro del país y que a la vez no sean competitivas a nivel mundial. Los alimentos no son los únicos sectores sensibles. Sectores industriales, especialmente si un país no es competitivo, también pueden serlo. En cuanto a mercancías como el sector textil, en donde gran parte de los costos de producción son referentes a la mano de obra, los países en desarrollo o PMA, por lo general, son más competitivos que los países desarrollados. Por lo tanto, los países desarrollados, para evitar que la producción nacional interna de estos sectores sea desplazada, aplican aranceles elevados.

- Mercancías producidas por industrias políticamente influyentes. En algunas ocasiones, los productores nacionales son competitivos; pero tienen un gran peso político en el país, es decir, hay industrias que pueden influir grandemente en las políticas comerciales del país. De esta manera, hay sectores que serán protegidos a pesar de que carezcan de necesidad.

Utilizando las características anteriores, la tabla 11 detalla la lista de productos sensibles y las razones por las cuales son considerados como sensibles para el caso de Costa Rica: 


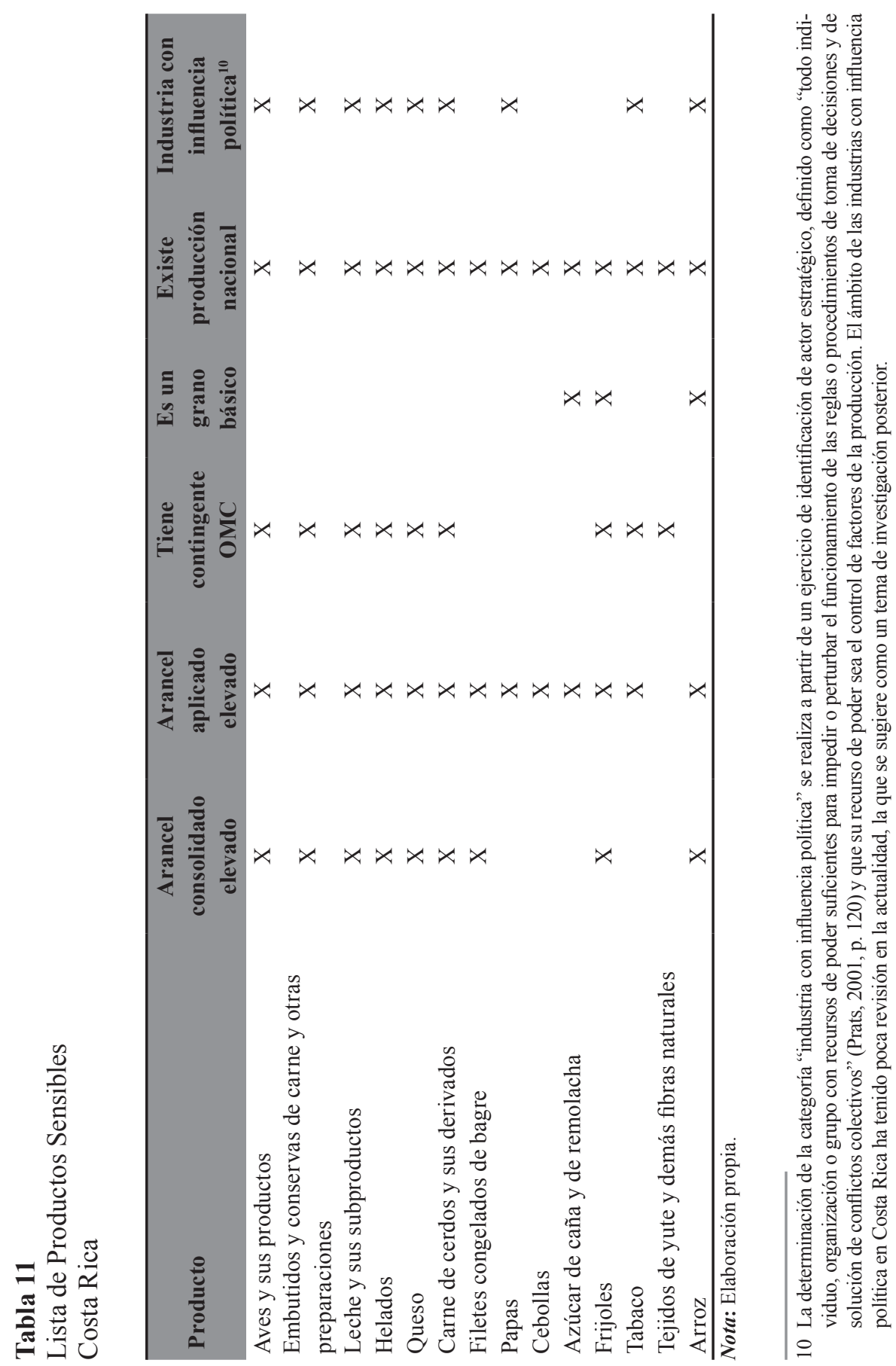


Construida y analizadala tabla 11, resulta pertinente validarla a propósito de lo que señalan Krugman, Olney y Wells, quienes detallan que:

En la actualidad del comercio internacional de bienes manufacturados está sujeto a aranceles bajos y a contingentes de importación relativamente escasos; la ropa es la principal excepción. Los productos agrícolas están sujetos a más restricciones, lo que refleja el poder de los agricultores en los países desarrollados. (Krugman, Olney y Wells, 2008, p. 348)

En efecto, los productos agropecuarios -como ya se indicó anteriormente- y los textiles en Costa Rica entran dentro de la categoría de mercancías sensibles al cumplir con las características dadas. Desde el punto de vista arancelario, lo principal es que la mayoría tiene elevados aranceles consolidados y aplicados y además tiene contingentes.

Las mercancías sensibles, paradójicamente, obtuvieron una mayor protección resultado del GATT. Este acuerdo pretendía favorecer a los PeD y los PmA, que en un inicio no tenían que hacer concesiones recíprocas desde el punto de vista arancelario. El resultado de ello es que los $\mathrm{PeD}$ conservaran aranceles elevados -puesto que no tenían aranceles recíprocos- en mercancías sensibles. En palabras del economista Jagdish Bhagwati "al tener que ampliar automáticamente las concesiones comerciales a los países en desarrollo sin exigirles reciprocidad, las economías avanzadas 'arreglaron' el problema con un sesgo en la selección de productos: solo redujeron los aranceles de los productos que les interesaban" (2013, p. 10). Esta práctica, como se demostró colateralmente en la investigación, ha sido recurrente también en economías de PeD y PmA -como es el caso de Costa Rica-. En general, los miembros del sistema multilateral de comercio no adquieren compromisos en cuanto a consolidación y aplicación de aranceles ni supresión total de contingentes en mercancías sensibles.

\section{CONCLUSIONES}

Los aranceles han sido, son y seguirán siendo un elemento importante que impacta al comercio entre los distintos interlocutores comerciales. Dentro de la liberalización de los aranceles, los países han recurrido a mecanismos multilaterales como la OMC y plurilaterales y bilaterales como lo son los TLC. De hecho, los TLC son instrumentos cada vez más empleados por los países para la apertura comercial.

Ahora bien, con el paso del tiempo, se ha demostrado que los temas arancelarios han perdido terreno, mientras otros elementos más profundos demuestran que impactan más el comercio que los propios aranceles. 
Antes de la Ronda Kennedy -dentro del marco del GATT- correspondiente a la sexta de 8 rondas en total antes de la creación de la $\mathrm{OMC}$, lo único que se negociaba eran temas netamente arancelarios. Fue después de la Ronda Kennedy en que se negociaron otros temas, lo cual demostró que, efectivamente, en las 5 rondas anteriores se disminuyeron sustancialmente los aranceles y los países apostaron a introducir nuevos temas que afectan el comercio de mercancías y servicios.

El arancel promedio mundial aplicado es de un 4\%, mientras que el arancel promedio aplicado por Costa Rica es de un 5,57\%. Con ello se demuestra que el arancel promedio aplicado de Costa Rica es bastante cercano al promedio mundial.

Según las estadísticas mundiales, solo un $2 \%$ del comercio mundial se realiza bajo más de un $10 \%$ de preferencia arancelaria. Esto quiere decir que el beneficio relativo de las preferencias arancelarias es bajo, ya que muchas economías tienden sus aranceles a $0 \%$.

En esta misma lógica, Costa Rica tiene vigentes TLC con 15 de los primeros 20 países de los que importa. Por otra parte, Costa Rica tiene vigentes TLC con 16 de los 20 países a los que más exporta. Ahora bien, se demuestra con ello que Costa Rica busca trato preferencial con sus principales socios comerciales, lo que no quiere decir que los flujos comerciales de las relaciones con estos interlocutores se dan efectivamente dentro de trato preferencial. La anterior es una interrogante a la que se buscará dar respuesta en posteriores investigaciones.

Una de las conclusiones más clarificadoras consiste en que la mayoría de los productos sensibles de Costa Rica cumplen tres o más de las siguientes características: tener un arancel consolidado elevado, tener un arancel aplicado elevado, contar con contingentes $\mathrm{OMC}$, ser un grano básico, existir una producción nacional o que su industria tenga influencia política.

Los productos sensibles son, en su mayoría, bienes agropecuarios; eso se demuestra con la existencia de contingentes y en la relación entre aranceles consolidados y aplicados. Todos los contingentes que tiene Costa Rica -a nivel OMC o dentro de los TLC- son de bienes agropecuarios.

Por otra parte, es fácilmente perceptible la diferencia en aranceles consolidados y aplicados entre bienes no agropecuarios y agropecuarios. Casi un 55\% de las líneas arancelarias de productos no agropecuarios tienen un arancel aplicado de $0 \%$ y no tienen ninguna línea arancelaria por encima de un arancel aplicado del 15\%; mientras que casi un 50\% de las líneas arancelarias de 
productos agropecuarios tienen un arancel aplicado igual o superior al $10 \%$, alcanzan, incluso, algunas líneas por encima del 100\% del arancel aplicado.

En el caso de productos sensibles -en su mayoría agropecuarios, con las características supra descritas-, se demuestra que los aranceles continúan siendo una medida proteccionista bastante utilizada por Costa Rica. El acceso a un arancel preferencial o a una cuota en una mercancía sensible puede ser motivo para la negociación de un acuerdo comercial.

Dentro de la agenda de investigación futura, a partir de haber identificado y corroborado las mercancías sensibles para el caso de Costa Rica, se pretenden realizar otras investigaciones donde se aborden las razones por las cuales Costa Rica negocia TLC (partiendo de que lo arancelario no sería la única motivación para la apertura por medio de acuerdos comerciales), en donde el análisis en torno a mercancías sensibles sería trascendente. Adicionalmente, también se espera continuar indagando si Costa Rica sigue las tendencias mundiales en cuanto a la negociación de acuerdos comerciales, tendencias que en el pasado la $\mathrm{OMC}$ ha recopilado. 


\section{REFERENCIAS}

Acosta Roca, F. (2005). Glosario de Comercio Exterior. México D.F.: ISEF.

Bhagwati, J. (2013). Amanece un nuevo sistema. Revista Finanzas y Desarrollo, $50(4), 8-13$.

Centrum voor Onderwijs Mondiaal (COM). (2010). Glosario. CMO. Recuperado de http://www.cmo.nl/epa-es/index.php/inicio/glosario

Giler Mejía, G., López López, E., Vargas Sánchez, M. y Romero, M. (2010). Análisis de la aplicación de barreras arancelarias a las importaciones ecuatorianas: caso textil y calzado. Guayaquil. Escuela Superior Politécnica del Litoral. Disponible en http://www.dspace.espol.edu.ec/handle/123456789/13313 (01 ene. 2015). Krugman, P., Olney, M. y Wells. R. (2008). Fundamentos de economía. Barcelona: Editorial Reverté.

Ministerio de Comercio Exterior (COMEX). (2014). Asignación de contingentes arancelarios. San José: COMEX.

Ministerio de Comercio Exterior (COMEX). (2015). Importaciones, estadísticas. San José. COMEX. Recuperado de http://www.comex.go.cr/estadisticas/importaciones.aspx

Organización Mundial de Aduanas (OMA). (s. f.). Normas de origen, manual. Bruselas: OMA. Recuperado de http://www.wcoomd.org/en/topics/origin/overview/ /media/304ABD35B23744218E65596E66DFF012.ashx

Organización Mundial de Comercio (OMC). (1994). Acuerdo general sobre aranceles aduaneros y comercio (1947). Ginebra: OMC.

Organización Mundial de Comercio (OMC). (2011a). Entender la OMC. Ginebra: OMC.

Organización Mundial de Comercio (OMC). (2011b). Informe sobre el comercio mundial 2011, La OMC y los acuerdos comerciales preferenciales: De la coexistencia a la coherencia. Ginebra: OMC.

Organización Mundial de Comercio (OMC). (2015a). Función de descarga de aranceles. Ginebra: OMC. Recuperado de http://tariffdata.wto.org/ReportersAndProducts.aspx

Organización Mundial de Comercio (OMC). (2015b). Información por Miembro, Costa Rica y la OMC. Ginebra: OMC. Recuperado de http://www.wto.org/ spanish/thewto_s/countries_s/costa_rica_s.htm

Organización Mundial de Comercio (OMC). (2015c). Tariff Profiles Costa Rica. Ginebra: OMC. Recuperado de http://stat.wto.org/TariffProfiles/CR_s.htm 
Organización Mundial de Comercio (OMC). (2016). Acuerdos comerciales regionales. Ginebra: OMC. Recuperado de http://www.wto.org/spanish/tratop_s/ region_s/region_s.htm

Prats i Català, J. (2001). Gobernabilidad democrática para el desarrollo humano. Marco conceptual y analítico. Revista Instituciones y Desarrollo, 10, 103-148.

Resolución No 53-96 (COMRIEDE VII). (17 de diciembre de 1996). Resolución No 53-96.

Secretaría de Integración Económica Centroamericana (SIECA). (2009). Estado de situación de la integración económica centroamericana. Guatemala: SIECA. Recuperado de http://www19.iadb.org/intal/intalcdi/PE/2009/02645.pdf 